\title{
An attentive neural architecture for joint segmentation and parsing and its application to real estate ads
}

\author{
Giannis Bekoulis*, Johannes Deleu, Thomas Demeester, Chris Develder \\ Ghent University - imec, IDLab, Department of Information Technology, \\ Technologiepark Zwijnaarde 15, 9052 Ghent, Belgium
}

\begin{abstract}
In processing human produced text using natural language processing (NLP) techniques, two fundamental subtasks that arise are (i) segmentation of the plain text into meaningful subunits (e.g., entities), and (ii) dependency parsing, to establish relations between subunits. Such structural interpretation of text provides essential building blocks for upstream expert system tasks: e.g., from interpreting textual real estate ads, one may want to provide an accurate price estimate and/or provide selection filters for end users looking for a particular property - which all could rely on knowing the types and number of rooms, etc. In this paper we develop a relatively simple and effective neural joint model that performs both segmentation and dependency parsing together, instead of one after the other as in most state-of-the-art works. We will focus in particular on the real estate ad setting, aiming to convert an ad to a structured description, which we name property tree, comprising the tasks of (1) identifying important entities of a property (e.g., rooms) from classifieds and (2) structuring them into a tree format. In this work, we propose a new joint model that is able to tackle the two tasks simultaneously and construct the property tree by (i) avoiding the error propagation that would arise from the subtasks one after the other in a pipelined fashion, and (ii) exploiting the interactions between the subtasks. For this purpose, we perform an extensive comparative study of the pipeline methods and the new proposed
\end{abstract}

\footnotetext{
* Corresponding author

Email addresses: giannis.bekoulis@ugent.be (Giannis Bekoulis),

johannes.deleu@ugent.be (Johannes Deleu), thomas.demeester@ugent.be (Thomas Demeester), chris.develdereugent. be (Chris Develder)
} 
joint model, reporting an improvement of over three percentage points in the overall edge $F_{1}$ score of the property tree. Also, we propose attention methods, to encourage our model to focus on salient tokens during the construction of the property tree. Thus we experimentally demonstrate the usefulness of attentive neural architectures for the proposed joint model, showcasing a further improvement of two percentage points in edge $F_{1}$ score for our application. While the results demonstrated are for the particular real estate setting, the model is generic in nature, and thus could be equally applied to other expert system scenarios requiring the general tasks of both (i) detecting entities (segmentation) and (ii) establishing relations among them (dependency parsing). Keywords: neural networks, joint model, relation extraction, entity recognition, dependency parsing

\section{Introduction}

Many consumer-oriented digital applications rely on input data provided by their target audience. For instance, real estate websites gather property descriptions for the offered classifieds, either from realtors or from individual sellers. In such cases, it

5 is hard to strike the right balance between structured and unstructured information: enforcing restrictions or structure upon the data format (i.e., predefined form) may reduce the amount or diversity of the data, while unstructured data (i.e., raw text) may require non-trivial (i.e., hard to automate) transformation to a more structured form to be useful/practical for the intended application. In the real estate domain, textual advertisements are an extremely useful but highly unstructured way of representing real estate properties. However, structured descriptions of the advertisements are very helpful, e.g., for real estate agencies to suggest the most appropriate sales/rentals for their customers, while keeping human reading effort limited. For example, special search filters, which are usually used by clients, cannot be directly applied to textual advertisements. On the contrary, a structured representation of the property (e.g., a tree format of the property) enables the simplification of the unstructured textual information by applying specific filters (e.g., based on the number of bedrooms, number of floors, or the requirement of having a bathroom with a toilet on the first floor), and it also ben- 
efits other related applications such as automated price prediction (Pace et al., 2000; Nagaraja et al., 2011).

The new real estate structured prediction problem as defined by Bekoulis et al. (2017) has as main goal to construct the tree-like representation of the property (i.e., the property tree) based on its natural language description. This can be approached as a relation extraction task by a pipeline of separate subtasks, comprising (i) named entity recognition (NER) (Nadeau \& Sekine, 2007) and (ii) relation extraction (Bach \& Badaskar, 2007). Unlike previous studies (Li \& Ji, 2014; Miwa \& Bansal, 2016) on relation extraction, in the work of Bekoulis et al. (2017), the relation extraction module is replaced by dependency parsing. Indeed, the relations that together define the structure of the house should form a tree, where entities are part-of one another (e.g., a floor is 30 part-of a house, a room is part-of a floor). This property tree is structurally similar to a parse tree. Although the work of Bekoulis et al. (2017) is a step towards the construction of the property tree, it follows a pipeline setting, which suffers from two serious problems: (i) error propagation between the subtasks, i.e., NER and dependency parsing, and (ii) cross-task dependencies are not taken into account, e.g., terms indicating relations (includes, contains, etc.) between entities that can help the NER module are neglected. Due to the unidirectional nature of stacking the two modules (i.e., NER and dependency parsing) in the pipeline model, there is no information flowing from the dependency parsing to the NER subtask. This way, the parser is not able to influence the predictions of the NER. Other studies on similar tasks (Li \& Ji, 2014, Kate \&

40 Mooney, 2010) have considered the two subtasks jointly. They simultaneously extract entity mentions and relations between them usually by implementing a beam-search on top of the first module (i.e., NER), but these methods require the manual extraction of hand-crafted features. Recently, deep learning with neural networks has received much attention and several approaches (Miwa \& Bansal, 2016, Zheng et al., 2017) 45 apply long short-term memory (LSTM) recurrent neural networks and convolutional neural networks (CNNs) to achieve state-of-the-art performance on similar problems. Those models rely on shared parameters between the NER and relation extraction components, whereby the NER module is typically pre-trained separately, to improve the training effectiveness of the joint model. 
In this work, we propose a new joint model to solve the real estate structured prediction problem. Our model is able to learn the structured prediction task without complicated feature engineering. Whereas previous studies (Miwa \& Bansal, 2016, Zheng et al., 2017, Li et al. 2016, 2017) on joint methods focus on the relation extraction problem, we construct the property tree which comes down to solving a dependency parsing problem, which is more constrained and hence more difficult. Therefore, previous methods are not directly comparable to our model and cannot be applied to our real estate task out-of-the-box. In this work, we treat the two subtasks as one by reformulating them into a head selection problem (Zhang et al., 2017).

This paper is a follow-up work of Bekoulis et al. (2017). Compared to the conference paper that introduced the real estate extraction task and applied some basic state-of-the-art techniques as a first baseline solution, we now introduce: (i) advanced neural models that consider the two subtasks jointly and (ii) modifications to the dataset annotation representations as detailed below. More specifically, the main contributions of this work are the following:

- We propose a new joint model that encodes the two tasks of identifying entities as well as dependencies between them, as a single head selection problem, without the need of parameter sharing or pre-training of the first entity recognition module separately. Moreover, instead of (i) predicting unlabeled dependencies and (ii) training an additional classifier to predict labels for the identified heads (Zhang et al. 2017), our model already incorporates the dependency label predictions in its scoring formula.

- We compare the proposed joint model against established pipeline approaches and report an $F_{1}$ improvement of $1.4 \%$ in the NER and $6.2 \%$ in the dependency parsing subtask, corresponding to an overall edge $F_{1}$ improvement of $3.4 \%$ in the property tree.

- Compared to our original dataset (Bekoulis et al. 2017), we introduce two extensions to the data: (i) we consistently assign the first mention of a particular entity in order of appearance in the advertisement as the main mention of the entity. This results in an $F_{1}$ score increase of about $3 \%$ and $4 \%$ for the joint and 
pipeline models, respectively. (ii) We add the equivalent relation to our annotated dataset to explicitly express that several mentions across the ad may refer to the same entity.

- We perform extensive analysis of several attention mechanisms that enable our LSTM-based model to focus on informative words and phrases, reporting an improved $F_{1}$ performance of about $2.1 \%$.

The rest of the paper is structured as follows. In Section 2 , we review the related work. Section 3 defines the problem and in Section 4, we describe the methodology followed throughout the paper and the proposed joint model. The experimental results are reported in Section 5 Finally, Section 6 concludes our work.

\section{Related work}

The real estate structured prediction problem from textual advertisements can be broken down into the sub-problems of (i) sequence labeling (identifying the core parts of the property) and (ii) non-projective dependency parsing (connecting the identified parts into a tree-like structure) (Bekoulis et al., 2017). One can address these two steps either one by one in a pipelined approach, or simultaneously in a joint model. The pipeline approach is the most commonly used approach (Bekoulis et al., 2017, Fundel et al. 2007, Gurulingappa et al. 2012, , treating the two steps independently and propagating the output of the sequence labeling subtask (e.g., named entity recognition) (Chiu \& Nichols, 2016, Lample et al., 2016) to the relation classification module (dos Santos et al., 2015, Xu et al., 2015). Joint models are able to simultaneously extract entity mentions and relations between them ( $\mathrm{Li} \& \mathrm{Ji}, 2014$, , Miwa \& Bansal, 2016). In this work, we propose a new joint model that is able to recover the tree-like structure of the property and frame it as a dependency parsing problem, given the non-projective tree structure we aim to output. We now present related works for the sequence labeling and dependency parsing subtasks, as well as for the joint models. 


\subsection{Sequence labeling}

Structured prediction problems become challenging due to the large output space. Specifically in NLP, sequence labeling (e.g., NER) is the task of identifying the entity mention boundaries and assigning a categorical label (e.g., POS tags) for each

Dependency parsing is a well studied task in the NLP community, which aims to analyze the grammatical structure of a sentence. We approach the problem of the property tree construction as a dependency parsing task i.e., to learn the dependency arcs of the classified. There are two well-established ways to address the dependency parsing 135 namely Hidden Markov Models (HMMs) (Rabiner \& Juang, 1986), Conditional Random Fields (CRFs) (Lafferty et al. 2001), Maximum Margin Markov Network $\left(\mathrm{M}^{3} \mathrm{~N}\right)$ (Taskar et al., 2003), generalized support vector machines for structured output (SVM ${ }^{\text {struct }}$ ) (Tsochantaridis et al. 2004) and Search-based Structured Prediction (SEARN) (Daumé III

et al. 2009). Those methods heavily rely on hand-crafted features and an in-depth review can be found in Nguyen \& Guo (2007). Several variations of these models that also require manual feature engineering have been used in different application settings „e.g., biology, social media context) and languages (e.g., Turkish) (Jung, 2012; Küçük \& Yazic1, 2012, Atkinson \& Bull, 2012, Konkol et al., 2015). Recently, deep learning with neural networks has been succesfully applied to NER. Collobert et al.(2011) proposed to use a convolutional neural network $(\mathrm{CNN})$ followed by a CRF layer over a sequence of word embeddings. Recurrent Neural Networks (RNNs) constitute another neural network architecture that has attracted attention, due to the state-of-the-art performance in a series of NLP tasks (e.g., sequence-to-sequence (Sutskever et al. 2014), 125 parsing (Kiperwasser \& Goldberg, 2016)). In this context, Gillick et al. (2016) use a sequence-to-sequence approach for modeling the sequence labeling task. In addition, several variants of combinations between LSTM and CRF models have been proposed (Lample et al., 2016, Huang et al., 2015, Ma \& Hovy, 2016) achieving state-of-the-art performance on publicly available datasets.

\subsection{Dependency parsing} problem, via graph-based and transition-based parsers. identified entity in the sentence. A number of different methods have been proposed, 
Graph-based: In the work of McDonald et al. (2005); McDonald \& Pereira (2007) dependency parsing requires the search of the highest scoring maximum spanning tree in graphs for both projective (dependencies are not allowed to cross) and non-projective (crossing dependencies are allowed) trees with the Eisner algorithm (Eisner, 1996) and the Chu-Liu-Edmonds algorithm (Chu \& Liu, 1965; Edmonds, 1967) respectively. It was shown that exploiting higher-order information (e.g., siblings, grand-parental relation) in the graph, instead of just using first-order information (i.e., parent relations) (Carreras, 2007; Zhang \& McDonald, 2012) may yield significant improvements of the parsing accuracy but comes at the cost of an increased model complexity. Koo et al. (2007) made an important step towards globally normalized models with handcrafted features, by adapting the Matrix-Tree Theorem (MTT) (Tutte, 2001) to train over all non-projective dependency trees. We explore an MTT approach as one of the pipeline baselines. Similar to recent advances in neural graph-based parsing (Zhang et al., 2017; Kiperwasser \& Goldberg, 2016, Wang \& Chang, 2016), we use LSTMs to capture richer contextual information compared to hand-crafted feature based methods. Our work is conceptually related to Zhang et al. (2017), who formulated the dependency parsing problem as a head selection problem. We go a step further in that direction, in formulating the joint parsing and labeling problem in terms of selecting the most likely combination of head and label.

155 Transition-based: Transition-based parsers (Yamada \& Matsumoto, 2003, Nivre et al., 2006) replace the exact inference of the graph-based parsers by an approximate but faster inference method. The dependency parsing problem is now solved by an abstract state machine that gradually builds up the dependency tree token by token. The goal of this kind of parsers is to find the most probable transition sequence from an initial to some terminal configuration (i.e., a dependency parse tree, or in our case a property tree) given a permissible set of actions (i.e., LEFT-ARC, RIGHT-ARC, SHIFT) and they are able to handle both projective and non-projective dependencies (Nivre, 2003, 2009). In the simplest case (i.e., greedy inference), a classifier predicts the next transition based on the current configuration. Compared to graph-based dependency parsers, transition-based parsers are able to scale better due to the linear time complexjty while graph-based complexity rises to $O\left(n^{2}\right)$ in the non-projective case. Chen \& 
Manning (2014) proposed a way of learning a neural network classifier for use in a greedy, transition-based dependency parser while using low-dimensional, dense word embeddings, without the need of manually extracting features. Globally normalized transition-based parsers (Andor et al., 2016) can be considered an extension of Chen \& Manning (2014), as they perform beam search for maintaining multiple hypotheses and introduce global normalization with a CRF objective. Dyer et al. (2015) introduced the stack-LSTM model with push and pop operations which is able to learn the parser transition states while maintaining a summary embedding of its contents. Although transition-based systems are well-known for their speed and state-of-the-art performance, we do not include them in our study due to their already reported poor performance in the real estate task (Bekoulis et al. 2017) compared to graph-based parsers.

\subsection{Joint learning}

Adopting a pipeline strategy for the considered type of problems has two main drawbacks: (i) sequence labeling errors propagate to the dependency parsing step, e.g., an incorrectly identified part of the house (entity) could get connected to a truly existing entity, and (ii) interactions between the components are not taken into account (feedback between the subtasks), e.g., modeling the relation between two potential entities may help in deciding on the nature of the entities themselves. In more general relation extraction settings, a substantial amount of work (Kate \& Mooney, 2010, Li \& Ji, 2014, Miwa \& Sasaki, 2014) jointly considered the two subtasks of entity recognition and relation extraction. However, all of these models make use of hand-crafted features that: (i) require manual feature engineering, (ii) generalize poorly between various applications and (iii) may require a substantial computational cost.

Recent advances on joint models for general relation extraction consider the joint task using neural network architectures like LSTMs and CNNs (Miwa \& Bansal, 2016, Zheng et al. 2017, Li et al., 2017). Our work is however different from a typical relation extraction setup in that we aim to model directed spanning trees, or, equivalently, non-projective dependency structures. In particular, the entities involved in a relation are not necessarily adjacent in the text since other entities may be mentioned in be- 


\begin{tabular}{lll}
\hline Entity type & Description & Examples \\
\hline property & The property. & bungalow, apartment \\
floor & A floor in a building. & ground floor \\
space & A room within the building. & bedroom, bathroom \\
subspace & A part of a room. & shower, toilet \\
field & $\begin{array}{l}\text { An open space inside or outside } \\
\text { the building. }\end{array}$ & bbq, garden \\
extra building & $\begin{array}{l}\text { An additional building which is } \\
\text { also part of the property. }\end{array}$ & garden house \\
&
\end{tabular}

Table 1: Real estate entity types.

tween, which complicates parsing. Indeed, in this work we focus on dependency parsing due to the difficulty of establishing the tree-like structure instead of only relation extraction (where each entity can have arbitrary relation arcs, regardless of other entities and their relations), which is the case for previously cited joint models. Moreover, unlike most of these works that frame the problem as a stacking of the two components, or at least first train the NER module to recognize the entities and then further train together with the relation classification module, we include the NER directly inside the dependency parsing component.

In summary, the conceptual strengths of our joint segmentation and dependency parsing approach (described in detail in Section 4 ) will be the following: compared to state-of-the-art joint models in relation extraction, it (i) is generic in nature, without requiring any manual feature engineering, (ii) extracts a complete tree structure rather than a single binary relation instance.

\section{Problem definition}

In this section, we define the specific terms that are used in our real estate structured prediction problem. We define an entity as an unambiguous, unique part of a property with independent existence (e.g., bedroom, kitchen, attic). An entity mention is defined as one or more sequential tokens (e.g., "large apartment") that can be potentially linked to one or more entities. An entity mention has a unique semantic meaning and refers to a specific entity, or a set of similar entities (e.g., "six bedrooms"). An entity itself is 
ment, skip and equivalent. The part-of relation is similar to the way that it was defined in the pipeline setting but instead of examining entities, i.e., sequences of tokens (e.g., "living room"), we examine if a (individual) token is part-of another (individual) token (e.g., "room" is part-of the "apartment"). We encode the entity identification task with the segment label and we follow the same approach as in the part-of relationships for the joint model. Specifically, we examine if a token is a segment of another token (e.g., the token "room" is attached as a segment to the token "living", "3" is attached as a segment to the token "bedrooms" and "spacious" is also attached as a segment to the token "bedrooms" - this way we are able to encode the segment "3 spacious bedrooms"). By doing so, we cast the sequence labeling subtask to a dependency parsing problem. The tokens that are referring to the same entity belong to the equivalent relation ("home" is equivalent to "apartment"). For each entity, we define the first mention in order of appearance in the text as main mention and the rest as equivalent to this main mention. Finally, each token that does not have any of the aforementioned types of relations has a skip relation with itself (e.g., "includes" has a skip relation with "includes"), such that each token has a uniquely defined head.

Thus, we cast the structured prediction task of extracting the property tree from the ad as a dependency parsing problem, where (i) an entity can be part-of only one (other) entity, because the decisions are taken simultaneously for all part-of relations (e.g., a certain room can only be part-of a single floor), and (ii) there are a priori no restrictions on the type of entities or tokens that can be part-of others (e.g., a room can be either part-of a floor, or the property itself, like an apartment). It is worth mentioning that dependency annotations for our problem exhibit a significant number of non-projective $\operatorname{arcs}(26 \%)$ where part-of dependencies are allowed to cross (see Fig. 3), meaning that entities involved in the part-of relation are non-adjacent (i.e., interleaved by other entities). For instance, all the entities or the tokens for the pipeline and the joint models, that are attached to the entity "garage" are overlapping with the entities that are attached to the entity "apartment", making parsing even more complicated: handling only projective dependencies as illustrated in Fig. 2 is an easier task. We note that the segment dependencies do not suffer from non-projectivity, since the tokens are always adjacent and sequential (e.g., "3 spacious bedrooms"). 


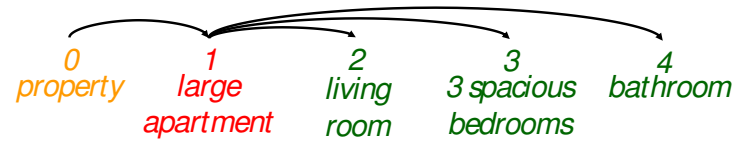

Figure 2: An example graph of projective part-of dependencies.

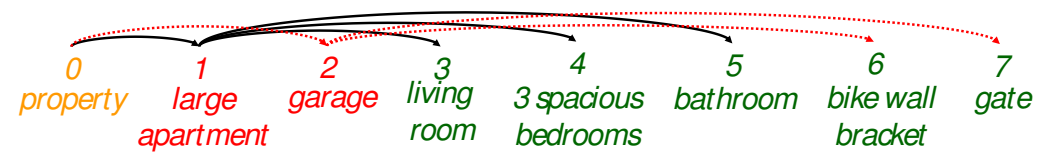

Figure 3: Graph representing the part-of dependencies of Fig. 1 The dashed arcs are representing the non-projective dependencies.

\section{Methodology}

We now describe the two approaches, i.e., the pipeline model and the joint model to construct the property tree of the textual advertisements, as illustrated in Fig. 4 For the pipeline system (Section 4.1), we (1) identify the entity mentions (Section 4.1.1), then (2) predict the part-of dependencies between them (Section 4.1.2), and finally (3) construct the tree representation (i.e., property tree) of the textual classified (e.g., as in Fig. 1). In step (2), we apply locally or globally trained graph-based models. We represent the result of step (2) as a graph model, and then solve step (3) by applying the maximum spanning tree algorithm (Chu \& Liu, 1965, Edmonds, 1967) for the directed case (see McDonald et al. (2005)). We do not apply the well-known and fast transition-based systems with hand-crafted features for non-projective dependency structures (Nivre, 2009: Bohnet \& Nivre, 2012), given the previously established poor performance thereof in Bekoulis et al. (2017). In Section 4.2, we describe the joint model where we perform steps (1) and (2) jointly. For step (3) we apply the maximum spanning tree algorithm (Chu \& Liu, 1965; Edmonds, 1967) similarly as in the pipeline setting (Section 4.1).

\subsection{Two-step pipeline}

Below we revisit the pipeline approach presented in Bekoulis et al. (2017), which serves as the baseline which we compare the neural models against. As mentioned before, the pipeline model comprises two subtasks: (1) the sequence labeling and the 
(2) part-of tree construction. In the following subsections, we describe the methods applied for both.

\subsubsection{Sequence labeling}

The first step in our pipeline approach is the sequence labeling subtask which is similar to NER. Assuming a textual real estate classified, we (i) identify the entity mention boundaries and (ii) map each identified entity mention to a categorical label, i.e., entity type. In general, in the sequence labeling tasks, it is beneficial to take into account the correlations between labels in adjacent tokens, i.e., consider the neighborhood, and jointly find the most probable chain of labels for the given input sentence (Viterbi algorithm for the most probable assignment). For instance, in our problem where we follow the NER standard BIO encoding (Ratinov \& Roth, 2009), the I-PROPERTY cannot be followed by I-SPACE without first opening the type by B-SPACE. We use a special case of the CRF algorithm (Lafferty et al., 2001, Peng \& McCallum, 2006), namely linear chain CRFs, which is commonly applied in the problem of sequence labeling to learn a direct mapping from the feature space to the output space (types) where we model label sequences jointly, instead of decoding each label independently. A linear-chain CRF with parameters $w$ defines a conditional probability $P_{w}(y \mid x)$ for the sequence of labels $y=y_{1}, \ldots, y_{N}$ given the tokens of the text advertisement $x=$ $x_{1}, \ldots, x_{N}$ to be

$$
P_{w}(y \mid x)=\frac{1}{Z(x)} \exp \left(w^{T} \phi(x, y)\right)
$$

where $Z$ is the normalization constant and $\phi$ is the feature function that computes a feature vector given the advertisement and the sequence of labels.

\subsubsection{Part-of tree construction}

The aim of the part-of tree construction subtask is to link each entity to its parent.

We approach the task as a dependency parsing problem but instead of connecting each token to its syntactical parent, we map only the entity set $I$ (e.g., "large villa", "3 spacious bedrooms") that has already been extracted by the sequence labeling subtask to a dependency structure $y$. Assuming the entity set $I=\left\{e_{0}, e_{1}, \ldots, e_{t}\right\}$ where $t$ is the number of identified entities, a dependency is a pair $(p, c)$ where $p \in I$ is the parent 


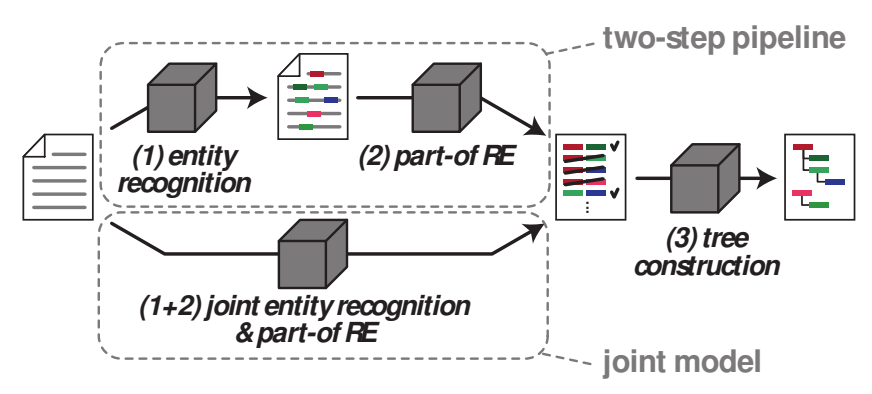

Figure 4: The full structured prediction system setup.

entity and $c \in I$ is the child entity. The entity $e_{0}$ is the dummy root-symbol that only appears as parent.

We will compare two approaches to predict the part-of relations: a locally trained model (LTM) scoring all candidate edges independently, versus a global model (MTT) which jointly scores all edges as a whole.

Locally trained model (LTM)

In the locally trained model (LTM), we adopt a traditional local discriminative method and apply a binary classification framework (Yamada \& Matsumoto, 2003) to learn the part-of relation model (step (2), based on standard relation extraction features such as the parent and child tokens and their types, the tokens in between, etc. For each candidate parent-child pair, the classifier gives a score that indicates whether it is probable for the part-of relation to hold between them. The output scores are then used for step (3), to construct the final property tree. Following McDonald et al. (2005); McDonald \& Pereira (2007), we view the entity set $I$ as a fully connected directed graph $G=\{V, E\}$ with the entities $e_{1}, \ldots, e_{t}$ as vertices $(V)$ in the graph $G$, and edges $E$ representing the part-of relations with the respective classifier scores as weights. One way to approach the problem is the greedy inference method where the predictions are made independently for each parent-child pair, thus neglecting that the global target output should form a property tree. We could adopt a threshold-based approach, i.e., keep all edges exceeding a threshold, which obviously is not guaranteed to end up with arc dependencies that form a tree structure (i.e., could even contain cycles). On the other hand, we can enforce the tree structure inside the (directed) graph by finding the 
maximum spanning tree. To this end, similar to McDonald et al. (2005); McDonald \& Pereira (2007), we apply the Edmonds' algorithm to search for the most probable non-projective tree structure in the weighted fully connected graph $G$.

\section{Globally trained model (MTT)}

The Matrix-Tree theorem (MTT) (Koo et al. 2007) is a globally normalized statistical method that involves the learning of directed spanning trees. Unlike the locally trained models, MTT is able to learn tree dependency structures, i.e., scoring parse trees for a given sentence. We use $D(I)$ to refer to all possible dependencies of the identified entity set $I$, in which each dependency is represented as a tuple $(h, m)$ in which $h$ is the head (or parent) and $m$ the modifier (or child). The set of all possible dependency structures for a given entity set $I$ is written $T(I)$. The conditional distribution over all dependency structures $y \in T(I)$ can then be defined as:

$$
P(y \mid I ; \theta)=\frac{1}{Z(I ; \theta)} \exp \left(\sum_{h, m \in y} \theta_{h, m}\right)
$$

in which the coefficients $\theta_{h, m} \in \mathbb{R}$ for each dependency $(h, m)$ form the real-valued weight vector $\theta$. The partition function $Z(I ; \theta)$ is a normalization factor that alas cannot be computed by brute-force, since it requires a summation over all $y \in T(I)$, containing an exponential number of possible dependency structures. However, an adaptation of the MTT allows us the direct and efficient computation of the partition function $Z(I ; \theta)$ as the determinant $\operatorname{det}(L(\theta))$ where $L(\theta)$ is the Laplacian matrix of the graph. It is worth mentioning that although MTT learns spanning tree structures during training, at the prediction phase, it is still required to use the maximum spanning tree algorithm (step (3) (McDonald et al. 2005; McDonald \& Pereira, 2007) as in the locally trained models.

\subsection{Joint model}

In this section, we present the new joint model sketched in Fig. 5, which simultaneously predicts the entities in the sentence and the dependencies between them, with the final goal of obtaining a tree structure, i.e., the property tree. We pose the problem of the identification of the entity mentions and the dependency arcs between them as a head 


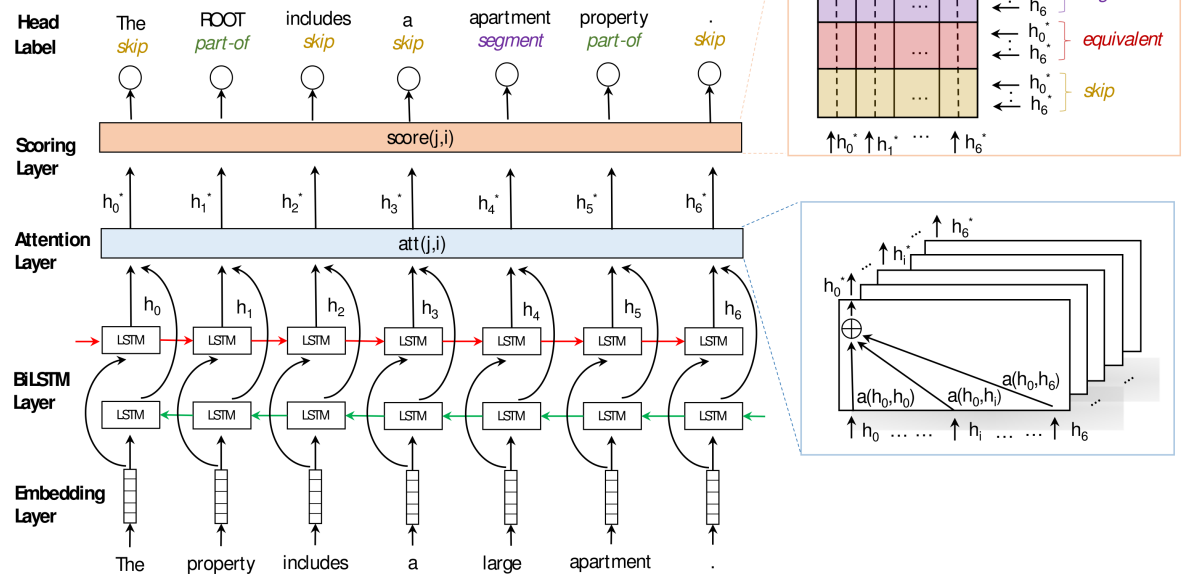

Figure 5: The architecture of the joint model.

selection problem (Zhang et al., 2017). Specifically, given as input a sentence of length $N$, the model outputs the predicted parent of each token of the advertisement and the most likely dependency label between them. We begin by describing how the tokens are represented in the model, i.e., with fixed pre-trained embeddings (Section 4.2.1), which form the input to an LSTM layer (Section 4.2.2). The LSTM outputs are used as input to the entity and dependency scoring layer (Section 4.2.3). As an extension of this model, we propose the use of various attention layers in between the LSTM and scoring layer, to encourage the model to focus on salient information, as described in Section 4.2.4. The final output of the joint model still is not guaranteed to form a tree structure. Therefore, we still apply Edmonds' algorithm (i.e., step (3) from the pipeline approach), described in Section 4.2 .5

\subsubsection{Embedding Layer}

The embedding layer maps each token of the input sequence $x_{1}, \ldots, x_{N}$ of the considered advertisement to a low-dimensional vector space. We obtain the word-level embeddings by training the Skip-Gram word2vec model (Mikolov et al. 2013) on a large collection of property advertisements. We add a symbol $x_{0}$ in front of the $N$-length input sequence, which will act as the root of the property tree, and is represented with 
an all-zeros vector in the embedding layer.

\subsubsection{Bidirectional LSTM encoding layer}

Many neural network architectures have been proposed in literature: LSTMs (Hochreiter \& Schmidhuber, 1997), CNNs (LeCun et al., 1989), Echo State Networks (Jaeger, 2001), or Stochastic Configuration Networks (Wang \& Li 2017), to name only a few. Many others can be found in reference works on the topic (Goodfellow et al., 2016, Goldberg \& Hirst, 2017). In this work, we use RNNs which have been proven to be particularly effective in a number of NLP tasks (Sutskever et al., 2014; Lample et al., 2016, Miwa \& Bansal, 2016). Indeed, RNNs are a common and reasonable choice to model sequential data and inherently able to cope with varying sequence lengths. Yet, plain vanilla RNNs tend to suffer from vanishing/exploding gradient problems and are hence not successful in capturing long-term dependencies (Bengio et al., 1994; Pascanu et al., 2013). LSTMs are a more advanced kind of RNNs, which have been successfully applied in several tasks to capture long-term dependencies, as they are able to effectively overcome the vanishing gradient problem. For many NLP tasks, it is crucial to represent each word in its own context, i.e., to consider both past (left) and future (right) neighboring information. An effective solution to achieve this is using a bidirectional LSTM (BiLSTM). The basic idea is to encode each sequence from left to right (forward) and from right to left (backward). This way, there is one hidden state which represents the past information and another one for the future information. The high-level formulation of an LSTM is:

$$
h_{i}, c_{i}=\operatorname{LSTM}\left(w_{i}, h_{i-1}, c_{i-1}\right), \quad i=0, \ldots, N
$$

where in our setup $w_{i} \in \mathbb{R}^{\tilde{d}}$ is the word embedding for token $x_{i}$, and with the input and states for the root symbol $x_{0}$ initialized as zero vectors. Further, $h_{i} \in \mathbb{R}^{d}$ and $c_{i} \in \mathbb{R}^{d}$ respectively are the output and cell state for the $i$ th position, where $d$ is the hidden state size of the LSTM. Note that we chose the word embedding size the same as the LSTM hidden state size, or $\tilde{d}=d$. The outputs from left to right (forward) are written as $\overrightarrow{h_{i}}$ and the outputs from the backwards direction as $\overleftarrow{h_{i}}$. The two LSTMs' outputs at 
position $i$ are concatenated to form the output $h_{i}$ at that position of the BiLSTM:

$$
h_{i}=\left[\overrightarrow{h_{i}} ; \overleftarrow{h_{i}}\right], \quad i=0, \ldots, N
$$

\section{(Znin} them jointly, instead of first obtaining binary predictions for unlabeled dependencies, followed by an additional classifier to predict the labels.

Given a text advertisement as a token sequence $x=x_{0}, x_{1}, \ldots, x_{N}$ where $x_{0}$ is the dummy root symbol, and a set $\mathcal{C}=\{$ part-of, segment, equivalent, skip $\}$ of predefined labels (as defined in Section 3), we aim to find for each token $x_{i}, i \in\{0, \ldots, N\}$ the most probable head $x_{j}, j \in\{0, \ldots, N\}$ and the most probable corresponding label $c \in$ $\mathcal{C}$. For convenience, we order the labels $c \in \mathcal{C}$ and identify them as $c_{k}, k \in\{0, \ldots, 3\}$. We model the joint probability of token $x_{j}$ to be the head of $x_{i}$ with $c_{k}$ the relation between them, using a softmax:

$$
P\left(\text { head }=x_{j}, \text { label }=c_{k} \mid x_{i}\right)=\frac{\exp \left(\operatorname{score}\left(h_{j}, h_{i}, c_{k}\right)\right)}{\sum_{\tilde{j}, \tilde{k}} \exp \left(\operatorname{score}\left(h_{\tilde{j}}, h_{i}, c_{\tilde{k}}\right)\right.}
$$

where $h_{i}$ and $h_{j}$ are the BiLSTM encodings for words $x_{i}$ and $x_{j}$, respectively. For the scoring formula score $\left(h_{j}, h_{i}, c_{k}\right)$ we use a neural network layer that computes the relative score between position $i$ and $j$ for a specific label $c_{k}$ as follows:

$$
\operatorname{score}\left(h_{j}, h_{i}, c_{k}\right)=V_{k}^{T} \tanh \left(U_{k} h_{j}+W_{k} h_{i}+b_{k}\right)
$$

with trainable parameters $V_{k} \in \mathbb{R}^{l}, U_{k} \in \mathbb{R}^{l \times 2 d}, W_{k} \in \mathbb{R}^{l \times 2 d}, b_{k} \in \mathbb{R}^{l}$, and $l$ the layer width. As detailed in Section 5.1, we set $l$ to be smaller than $2 d$, similar to Dozat \& Manning (2017) due to the fact that training on superfluous information reduces the parsing speed and increases tendency towards overfitting. We train our model by 
minimizing the cross-entropy loss $\mathcal{L}$, written for the considered training instance as:

$$
\mathcal{L}=\sum_{i=0}^{N}-\log P\left(\text { head }=y_{i}, \text { label }=c_{i} \mid x_{i}\right)
$$

where $y_{i} \in x$ and $c_{i} \in \mathcal{C}$ are the ground truth head and label of $x_{i}$, respectively. After training, we follow a greedy inference approach and for each token, we simultaneously keep the highest scoring head $\hat{y}_{i}$ and label $\hat{c}_{i}$ for $x_{i}$ based on their estimated joint probability:

$$
\left(\hat{y_{i}}, \hat{c_{i}}\right)=\underset{x_{j} \in x, c_{k} \in \mathcal{C}}{\operatorname{argmax}} P\left(\text { head }=x_{j}, \text { label }=c_{k} \mid x_{i}\right)
$$

The predictions $\left(\hat{y}_{i}, \hat{c}_{i}\right)$ are made independently for each position $i$, neglecting that the final structure should be a tree. Nonetheless, as demonstrated in Section 5.2 , the highest scoring neural models are still able to come up with a tree structure for $78 \%$ of the ads. In order to ensure a tree output in all cases, however, we apply Edmonds' algorithm on the output.

\subsubsection{Attention Layer}

The attention mechanism in our structured prediction problem aims to improve the model performance by focusing on information that is relevant to the prediction of the most probable head for each token. As attention vector, we construct the new context vector $h_{i}^{*}$ as a weighted average of the BiLSTM outputs

$$
h_{j}^{*}=\sum_{i=0}^{N} a\left(h_{j}, h_{i}\right) h_{i}
$$

in which the coefficients $a\left(h_{j}, h_{i}\right)$, also called the attention weights, are obtained as follows:

$$
a\left(h_{j}, h_{i}\right)=\frac{\exp \left(\operatorname{att}\left(h_{j}, h_{i}\right)\right)}{\sum_{\tilde{i}=0}^{N} \exp \left(\operatorname{att}\left(h_{j}, h_{\tilde{i}}\right)\right)} .
$$

The attention function $a t t\left(h_{j}, h_{i}\right)$ is designed to measure some form of compatibility between the representation $h_{i}$ for $x_{i}$ and $h_{j}$ for $x_{j}$, and the attention weights $a\left(h_{j}, h_{i}\right)$ are obtained from these scores by normalization using a softmax function. In the following, we will describe in detail the various attention models that we tested with our joint model. 
Three commonly used attention mechanisms are listed in eqs. (11) to (13): the additive (Vinyals et al. 2015), bilinear, and multiplicative attention models (Luong et al., 2015), which have been extensively used in machine translation. Given the representations $h_{i}$ and $h_{j}$ for tokens $x_{i}$ and $x_{j}$, we compute the attention scores as follows:

$$
\begin{gathered}
a t t_{\text {additive }}\left(h_{j}, h_{i}\right)=V_{a} \tanh \left(U_{a} h_{j}+W_{a} h_{i}+b_{a}\right) \\
\operatorname{att}_{\text {bilinear }}\left(h_{j}, h_{i}\right)=h_{j}^{T} W_{\text {bil }} h_{i} \\
\operatorname{att}_{\text {multiplicative }}\left(h_{j}, h_{i}\right)=h_{j}^{T} h_{i}
\end{gathered}
$$

where $V_{a} \in \mathbb{R}^{l}, U_{a}, W_{a} \in \mathbb{R}^{l \times 2 d}, W_{b i l} \in \mathbb{R}^{2 d \times 2 d}$ and $b_{a} \in \mathbb{R}^{l}$ are learnable parameters of the model.

\section{Biaffine attention}

We use the biaffine attention model (Dozat \& Manning, 2017) which has been recently applied to dependency parsing and is a modification of the neural graph-based approach that was proposed by Kiperwasser \& Goldberg (2016). In this model, Dozat \& Manning (2017) tried to reduce the dimensionality of the recurrent state of the LSTMs by applying a such neural network layer on top of them. This idea is based on the fact that there is redundant information in every hidden state that (i) reduces parsing speed and (ii) increases the risk of overfitting. To address these issues, they reduce the dimensionality and apply a nonlinearity afterwards. The deep bilinear attention mechanism is defined as follows:

$$
\begin{gathered}
h_{i}^{\text {dep }}=V_{\text {dep }} \tanh \left(U_{\text {dep }} h_{i}+b_{\text {dep }}\right) \\
h_{j}^{\text {head }}=V_{\text {head }} \tanh \left(U_{\text {head }} h_{j}+b_{\text {head }}\right)
\end{gathered}
$$




$$
\operatorname{att}_{\text {biaffine }}\left(h_{j}^{\text {head }}, h_{i}^{\text {dep }}\right)=\left(h_{j}^{\text {head }}\right)^{T} W_{\text {bil }} h_{i}^{\text {dep }}+B h_{j}^{\text {head }}
$$

where $U_{\text {dep }}, U_{\text {head }} \in \mathbb{R}^{l \times 2 d}, V_{\text {dep }}, V_{\text {head }} \in \mathbb{R}^{p \times l}, W_{\text {bil }} \in \mathbb{R}^{p \times p}, B \in \mathbb{R}^{p}$ and $b_{\text {dep }}$,

\section{Tensor attention}

This section introduces the Neural Tensor Network (Socher et al., 2013) that has been used as a scoring formula applied for relation classification between entities. The task can be described as link prediction between entities in an existing network of relationships. We apply the tensor scoring formula as if tokens are entities, by the following function:

$$
\operatorname{att}_{\text {tensor }}\left(h_{j}, h_{i}\right)=U_{t} \tanh \left(h_{j}^{T} W_{t} h_{i}+V_{t}\left(h_{j}+h_{i}\right)+b_{t}\right)
$$

where $W_{t} \in \mathbb{R}^{2 d \times l \times 2 d}, V_{t} \in \mathbb{R}^{l \times 2 d}, U_{t} \in \mathbb{R}^{l}$ and $b_{t} \in \mathbb{R}^{l}$.

\section{Edge attention}

In the edge attention model, we are inspired by Gilmer et al. (2017), which applies neural message passing in chemical structures. Assuming that words are nodes inside the graph and the message flows from node $x_{i}$ to $x_{j}$, we define the edge representation to be:

$$
\operatorname{edge}\left(h_{j}, h_{i}\right)=\tanh \left(U_{e} h_{j}+W_{e} h_{i}+b_{e}\right)
$$

The edge attention formula is computed as:

$$
\operatorname{att}_{\text {edge }}\left(h_{j}, h_{i}\right)=\frac{1}{N}\left(A_{s r c} \sum_{\tilde{i}=0}^{N} \operatorname{edge}\left(h_{j}, h_{\tilde{i}}\right)+A_{d s t} \sum_{\tilde{j}=0}^{N} \operatorname{edge}\left(h_{\tilde{j}}, h_{i}\right)\right)
$$

where $U_{e}, W_{e} \in \mathbb{R}^{l \times 2 d}, A_{s r c}, A_{d s t} \in \mathbb{R}^{2 d \times l}$ and $b_{e} \in \mathbb{R}^{l}$. The source and destination matrices respectively encode information for the start to the end node, in the directed edge. Running the edge attention model for several times can be achieved by stacking the edge attention layer multiple times. This is known as message passing phase and we can run it for several $(T>1)$ time steps to obtain more informative edge representations. 
In this section, we present the experimental results of our study. We describe the dataset, the setup of the experiments and we compare the results of the methods analysed in the previous sections.

\subsection{Experimental setup}

ments from real estate agency websites. From this large dataset, a sub-collection of 2,318 classifieds have been manually annotated by 3 trained human annotators ( 1 annotation per ad, 773 ads per annotator). The annotations follow the format of the 
property tree that is described in detail in Section 3 and is illustrated in Fig. 1. The dataset is available for research purposes, see our github codebase ${ }^{1}$ In the experiments, we use only the annotated text advertisements for the pipeline setting, i.e., LTM (locally trained model), MTT (globally trained model). In the case of the neural network approach, we train the embeddings on the large collection by using the word2vec model (Mikolov et al. 2013) whereas in the joint learning, we use only the annotated documents, similar to the pipeline approach. The code of the LTM and the MTT handcrafted systems is available on github! ${ }^{1}$ We also use our own CRF implementation. The code for the joint model has been developed in Python with the Tensorflow machine learning library (Abadi et al. 2016) and will be made public as well. For the evaluation, we use $70 \%$ for training, $15 \%$ for validation and $15 \%$ as test set. We measure the performance by computing the $F_{1}$ score on the test set. The accuracy metric can be misleading in our case since we have to deal with imbalanced data (the skip label is over-represented). We only report numbers on the structured classes, i.e., segment and part-of since the other dependencies (skip, equivalent) are auxiliary in the joint models and do not directly contribute to the construction of the actual property tree. For the overall $F_{1}$, we are again only considering the structured classes. Finally, we report the number of property trees (which shows how likely our model is to produce trees without applying Edmonds' algorithm, i.e., by greedy inference alone) for all the models before applying Edmonds' algorithm that guarantees the tree structure of the predictions.

For the pipeline models, we train the $\mathrm{CRF}$ with regularization parameter $\lambda_{C R F}=$ 10 and the LTM and MTT with $C=1$ based on the best hyperparameters on the validation set. As binary classifier, we use logistic regression. For the joint model, we train 128-dimensional word2 $\mathrm{vec}$ embeddings on a collection of $887 \mathrm{k}$ advertisements. In general, using larger embeddings dimensions (e.g., 300), does not affect the performance of our models. We consistently used single-layer LSTMs through our experiments to keep our model relatively simple and to evaluate the various attention methods on top of that. We have also reported results on the joint model using a two-layer

1 https://github.com/bekou/ad_data 


\begin{tabular}{|c|c|c|c|c|c|c|c|c|c|}
\hline & \multirow[b]{2}{*}{ Model } & \multicolumn{2}{|c|}{ Precision } & \multicolumn{2}{|c|}{ Recall } & \multicolumn{3}{|c|}{$F_{1}(\%)$} & \multirow{2}{*}{$\begin{array}{c}\text { Trees } \\
(\% \text { of ads })\end{array}$} \\
\hline & & segment & part-of & segment & part-of & segment & part-of & Overall & \\
\hline \multirow{2}{*}{ 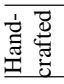 } & LTM & 73.77 & 60.53 & 70.98 & 60.40 & 72.35 & 60.47 & 64.76 & 37.18 \\
\hline & MTT & 73.77 & 61.15 & 70.98 & 61.01 & 72.35 & 61.08 & 65.15 & 43.23 \\
\hline \multirow{3}{*}{ 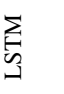 } & LSTM & 70.24 & 65.23 & 77.73 & 70.32 & 73.80 & 67.68 & 68.82 & 68.30 \\
\hline & LSTM+E & 70.18 & 63.92 & 77.77 & 71.08 & 73.78 & 67.31 & 68.57 & 68.30 \\
\hline & $2 \mathrm{xLSTM}+\mathrm{E}$ & 73.91 & 69.88 & 75.78 & 71.22 & 74.83 & 70.54 & 70.90 & 78.09 \\
\hline \multirow{8}{*}{ 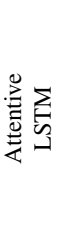 } & Additive & 72.97 & 65.71 & 76.45 & 70.90 & 74.67 & 68.21 & 69.46 & 74.35 \\
\hline & Bilinear & 70.25 & 66.34 & 79.96 & 72.53 & 74.79 & 69.29 & 70.20 & 72.62 \\
\hline & Multiplicative & 71.12 & 66.40 & 77.81 & 71.26 & 74.31 & 68.75 & 69.70 & 72.91 \\
\hline & Biaffine & 70.01 & 64.67 & 78.32 & 71.04 & 73.93 & 67.71 & 68.75 & 74.06 \\
\hline & Tensor & 71.53 & 64.68 & 76.17 & 70.79 & 73.78 & 67.60 & 68.68 & 69.16 \\
\hline & Edge $_{1}$ & 71.56 & 67.46 & 78.24 & 71.31 & 74.75 & 69.33 & 70.08 & 70.32 \\
\hline & Edge $_{2}$ & 72.03 & 66.09 & 75.35 & 70.99 & 73.65 & 68.46 & 69.12 & 73.48 \\
\hline & Edge $_{3}$ & 71.74 & 67.69 & 78.44 & 73.00 & 74.94 & 70.25 & 70.70 & 78.96 \\
\hline
\end{tabular}

Table 2: Performance of the three approaches on the structured prediction task. The top rows are for the pipeline approach, i.e., hand-crafted features. The next block of results presents the results for the neural joint model based on LSTMs. The bottom block contains results of the joint models augmented with several attentive architectures. Edmonds' algorithm is applied in all of the models to retain the tree structure, except for the LSTM joint model. The LSTM+E is the LSTM model with Edmonds' algorithm included. The $2 \mathrm{xLSTM}+\mathrm{E}$ is the same joint model but it simply uses a stack of two LSTM layers. In the experiments with attention, we use a one-stack LSTM. The rightmost column is the percentage of the ads that are valid trees before applying Edmonds' (i.e., step (3) of Fig. 4), showing the ability of the model to form trees during greedy inference. In the Edge $i$ models, the number $i$ stands for the number of times that we have run the message passing phase.

stacked LSTM joint model, although it needs a higher computation time compared to a single-layer LSTM with an attention layer on top. The hidden size of the LSTMs is $d=128$ and the size of the neural network used in the scoring and the attention layer is fixed to $l=32$. The optimization algorithm used is Adam (Kingma \& Ba, 2014) with a learning rate of $10^{-3}$. To reduce the effect of overfitting, we regularize our model using the dropout method (Srivastava et al. 2014). We fix the dropout rate on the input of the LSTM layer to 0.5 to obtain significant improvements $\left(\sim 1 \%-2 \% F_{1}\right.$ score increase, depending on the model). For the two-layer LSTM, we fix the dropout rate to 0.3 in each of the input layers since this leads to largest performance increase on the validation set. We have also explored gradient clipping without further improvement on our results. In the joint model setting, we follow the evaluation strategy of early stopping (Caruana et al., 2000; Graves et al. 2013) based on the performance of the validation set. In most of the experiments, we obtain the best hyperparameters after $\sim 60$ epochs. 


\subsection{Comparison of the pipeline and the joint model}

One of the main contributions of our study is the comparison of the pipeline approach and the proposed joint model. We formulated the problem of identifying the entities (i.e., segments) and predicting the dependencies between them (i.e., construction of the property tree) as a joint model. Our neural model, unlike recent studies (Miwa \& Bansal, 2016; Zheng et al. 2017) on joint models that use LSTMs to handle similar tasks, does not need two components to model the problem (i.e., NER and dependency parsing). To the best of our knowledge, our study is the first that formulates the task in an actual joint setting without the need to pre-train the sequence labeling component or for parameter sharing between them, since we use only one component for both subtasks. In Table 2 , we present the results of the pipeline model (hand-crafted) and the proposed joint model (LSTM). The improvement of the joint model over the pipeline is unambiguous, i.e., $3.42 \%$ overall $F_{1}$ score difference between MTT (highest scoring pipeline model) and LSTM+E (LSTM model with Edmonds' algorithm). An additional increase of $\sim 2.3 \%$ is achieved when we consider two-layer LSTMs ( $2 x \mathrm{~L}-$ STM+E) for our joint model. All results in Table 2, except for the LSTM, are presented using Edmonds' algorithm on top, to construct the property tree. Examining each label separately, we observe that the original LSTM+E model (73.78\%) performs better by $1.43 \%$ in entity segmentation than the CRF $(72.35 \%)$. The LSTM model achieves better performance in the entity recognition task since it has to learn the two subtasks simultaneously resulting in interactions between the components (i.e., NER and dependency parser). This way, the decisions for the entity recognition can benefit from predictions that are made for the part-of relations. Concerning the part-of dependencies, we note that the LSTMs outperform the hand-crafted approaches by $6.23 \%$. Also, the number of valid trees that are constructed before applying Edmonds' algorithm is almost twice as high for the LSTM models. Stacking two-layer LSTMs results in an additional $\sim 1 \%$ improvement in the segmentation task and $\sim 3 \%$ in the part-of relations. The greedy inference for the hand-crafted methods does not produce well-formed trees, meaning that post-processing with Edmonds' algorithm (enforce tree structure) is expected to increase the performance of the hand-crafted models compared to the LSTM model performance. Indeed, the performance of the feature based hand-crafted mod- 
els (i.e., LTM and MTT) without the Edmonds' on top is not reported in Table 2 due to their poor performance in our task (i.e., $\sim 60 \%$ overall $F_{1}$ and $\sim 51 \%$ for part-of), but after post-processing with Edmonds' the performance significantly increases (i.e., $\sim 65 \%$ ). On the other hand, applying the Edmonds' algorithm on the LSTM model leads to marginally decreased performance $(\sim 0.2 \%)$ compared to the original LSTM model, probably indicating that enforcing structural constraints is not beneficial for a model that clearly has the ability to form valid tree structures during greedy inference. Although one might be tempted not to enforce the tree structure (post-process with Edmonds'), due to the nature of our problem, we have to enforce tree constraints in all of the models.

\subsection{Comparison of the joint and the attention model}

After having established the superior performance of neural approach using LSTMs over the more traditional (LTM and MTT) methods based on hand-crafted features, we now discuss further improvements using attentive models. The attention mechanisms are designed to encourage the joint model to focus on informative tokens. We exploited several attention mechanisms as presented in Section 4.2.4. Table 2 shows the performance of the various models. Overall, the attention models are performing better in terms of overall $F_{1}$ score compared to the original joint model with the Edmonds' on top. Although the performance of the Biaffine and the Tensor models is limited compared to the improvement of the other attentive models, we focus on: (i) the Biaffine model since it achieved state-of-the-art performance on the dependency parsing task and (ii) the Tensor model because we were expecting that it would perform similarly to the Bilinear model (it has a bilinear tensor layer). Despite its simplicity, the Bilinear model is the second best performing attentive model in Table 2 in terms of overall $F_{1}$ score. Edge e $_{3}\left(70.70 \%\right.$ overall $F_{1}$ score) achieves better results than the other attention mechanisms in the entity recognition and in the dependency parsing tasks. We observe that running the message passing step multiple times in the Edge model, gives an increasing trend in the number of valid trees that were constructed before applying the maximum spanning tree algorithm. This is not surprising since we expect that running the message passing phase multiple times leads to improved edge representations. The 
maximum number of trees without post-processing by Edmonds' is attained when we run the message passing for 3 times whereas further increasing the number beyond 3 (e.g., 4) appears no longer beneficial. Stacking a second LSTM layer on top of the joint model $(2 \mathrm{xLSTM}+\mathrm{E})$ marginally improves the performance by $0.2 \%$ compared to the Edge $_{3}$ attention model. But adding a second LSTM layer comes with the additional cost of an increased computation time compared to the joint models with the attention layers on top. This illustrates that: (i) there might be some room for marginally improving the attention models even further, and (ii) we do not have to worry about the quadratic nature of our approach since in terms of speed the attentive models are able to surpass the two-layer LSTMs. The sequential processing of the LSTMs might be the reason that slows down the computation time for the 2xLSTM over the rest of the attentive models. Specifically, on an Intel(R) Xeon(R) CPU E5-2650 v2 @ 2.60GHz processor, the best performing model (i.e., $\mathrm{Edge}_{3}$ ) takes $\sim 2$ minutes per epoch while in the 2 XLSTM case, it takes $\sim 2.5$ minutes leading to a slowdown of $\sim 25 \%$. The percentage of the ads that are valid trees is $1 \%$ better in the Edge 3 over the two-layer LSTM showcasing the ability of the Edge model to form more valid trees during greedy inference.

\subsection{Discussion}

In this section, we discuss some additional aspects of our problem and the approaches that we follow to handle them. As we mentioned before, a single entity can be present in the text with multiple mentions. This brings an extra difficulty to our task. For instance, in the example of Fig. 1. the entity "large apartment" is expressed in the ad with the mentions "large apartment" and "home". Hence it is confusing to which mention the other entities should be attached to. One way would be to attach them to both and then eliminate one of the connections using Edmonds' spanning tree algorithm, which is the approach adopted in Bekoulis et al. (2017). The problematic issue with this approach is that the spanning tree algorithm would randomly remove all mentions but one, possibly resulting in uncertain outcomes. To avoid this problem, we now use as the main mention for an entity the first mention in order of appearance in the text (e.g., "large apartment" in our example) and the remaining mentions (e.g., "home") 
are attached as equivalent mentions to the main one. Usually, the most informative mention for an entity is the one that appears first, because we again refer to an entity mentioned before, often with a shorter description. Following our intuition, the neural model increases its overall performance by $~ 3 \%$ (from $66 \%$ to $69 \%$ and more than $5 \%$ in the part-of relation) and the pipeline approaches by almost $4 \%$ (from $61 \%$, reported in Bekoulis et al. (2017) to $65 \%$ and more than $5 \%$ in the part-of relation).

We also experimented with introducing the equivalent relations. Although it is a strongly under-represented class in the dataset and the model performs poorly for this label (an equivalent edge $F_{1}$ score of 10\%), introducing the equivalent label is the natural way of modeling our problem (i.e., assigning each additional mention as equivalent to the main mention). We find out that introducing this type of relation leads to a slight decrease $(\sim 1 \%)$ in the part-of and a marginal increase $(\sim 0.3 \%)$ in the segment relations which are the main relations while retaining the nature of our problem. In the pipeline approach, it results in an $9 \%$ drop in the $F_{1}$ score of the partof relation. This is the reason that the results as presented in Table 2 do not consider the equivalent relation for the hand-crafted model to make a fair comparison in the structured classes.

We believe our experimental comparison of the various architectural model variations provides useful findings for practitioners. Specifically, for applications requiring both segmentation (entity recognition) and dependency parsing (structured prediction), our findings can be qualitatively summarized as follows: (i) joint modeling is the most appropriate approach since it reduces error propagation between the components, (ii) the LSTM model is much more effective (than models relying on handcrafted features) because it automatically extracts informative features from the raw text, (iii) attentive models are proven effective because they encourage the model to focus on salient tokens, (iv) the edge attention model leads to an improved performance since it better encodes the information flow between the entities by using graph representations, and (v) stacking a second LSTM marginally increases the performance, suggesting that there might be some room for slight improvement of the attention models by adding LSTM layers.

Finally, we point out how exactly our model relates to state-of-the-art in the field. 
Our joint model is able to both extract entity mentions (i.e., perform segmentation)

and do dependency parsing, which we demonstrate on the real estate problem. Previous studies (Kate \& Mooney, 2010, Li \& Ji, 2014, Miwa \& Sasaki, 2014) that jointly considered the two subtasks (i.e., segmentation and relation extraction): (i) require manual feature engineering and (ii) generalize poorly between various applications. On the other hand, in our work, we rely on neural network methods (i.e., LSTMs) to automatically extract features from the real estate textual descriptions and perform the two tasks jointly. Although there are other methods which use neural network architectures (Miwa \& Bansal, 2016; Zheng et al., 2017, Li et al., 2017) that focus on the relation extraction problem, our work is different in that we aim to model directed spanning trees and thus to solve the dependency parsing problem which is more constrained and difficult (than extracting single instances of binary relations). Moreover, the cited methods require either parameter sharing or pre-training of the segmentation module, which complicates learning. Therefore, cited methods are not directly comparable to our model and cannot be applied to our real estate task out-of-the-box. However, our model's main limitation is the quadratic scoring layer that increases the time complexity of the segmentation task from linear (which is the complexity of a conditional random field, CRF) to $O\left(n^{2}\right)$. As a result, it sacrifices standard linear complexity of the segmentation task, in order to reduce the error propagation between the subtasks and thus perform learning in a joint, end-to-end differentiable, setting.

\section{Conclusions}

610 In this paper, we proposed an LSTM-based neural model to jointly perform segmentation and dependency parsing. We apply it to a real estate use case processing textual ads, thus (1) identifying important entities of the property (e.g., rooms) and (2) structuring them into a tree format based on the natural language description of the property. We compared our model with the traditional pipeline approaches that have been adapted to our task and we reported an improvement of $3.4 \%$ overall edge $F_{1}$ score. Moreover, we experimented with different attentive architectures and stacking of a second LSTM layer over our basic joint model. The results indicate that exploiting atten- 
tion mechanisms that encourage our model to focus on informative tokens, improves the model performance (increase of overall edge $F_{1}$ score with $\sim 2.1 \%$ ) and increases the ability to form valid trees in the prediction phase (4\% to $10 \%$ more valid trees for the two best scoring attention mechanisms) before applying the maximum spanning tree algorithm.

The contribution of this study to the research in expert and intelligent systems is three-fold: (i) we introduce a generic joint model, simultaneously solving both subtasks of segmentation (i.e., entity extraction) and dependency parsing (i.e., extracting relationships among entities), that unlike previous work in the field does not rely on manually engineered features, (ii) in particular for the real estate domain, extracting a structured property tree from a textual ad, we refine the annotations and additionally propose attention models, compared to initial work on this application, and finally (iii) we demonstrate the effectiveness of our proposed generic joint model with extensive experiments (see aforementioned $F_{1}$ improvement of $2.1 \%$ ). Despite the experimental focus on the real estate domain, we stress that the model is generic in nature, and could be equally applied to other expert system scenarios requiring the general tasks of both detecting entities (segmentation) and establishing relations among them (dependency parsing). We furthermore note that our model, rather than focusing on extracting a single binary relation from a sentence (as in traditional relation extraction settings), produces a complete tree structure.

Future work can evaluate the value of our joint model we introduced in other specific application domains (e.g., biology, medicine, news) for expert and intelligent systems. For example, the method can be evaluated for entity recognition and binary relation extraction (the ACE 04 and ACE 05 datasets; see Miwa \& Bansal (2016)) or in adverse drug effects from biomedical texts (see Li et al. (2016)). In terms of model extensions and improvements, one research issue is to address the time complexity of the NER part by modifying the quadratic scoring layer for this component. An additional research direction is to investigate different loss functions for the NER component (e.g., adopting a conditional random field (CRF) approach), since this has been proven effective in the NER task on its own (Lample et al., 2016). A final extension we envision is to enable multi-label classification of relations among entity pairs. 


\section{Acknowledgments}

Flanders Innovation \& Entrepreneurship (VLAIO).

\section{References}

Abadi, M., Agarwal, A., Barham, P., Brevdo, E., Chen, Z., Citro, C., Corrado, G. S., Davis, A., Dean, J., Devin, M. et al. (2016). TensorFlow: Large-scale machine learning on heterogeneous distributed systems. arXiv preprint arXiv:1603.04467, .

Andor, D., Alberti, C., Weiss, D., Severyn, A., Presta, A., Ganchev, K., Petrov, S., \& Collins, M. (2016). Globally normalized transition-based neural networks. In Proceedings of the 54th Annual Meeting of the Association for Computational Linguistics (Volume 1: Long Papers) (pp. 2442-2452). Berlin, Germany.

Atkinson, J., \& Bull, V. (2012). A multi-strategy approach to biological named entity recognition. Expert Systems with Applications, 39(17), 12968 - 12974. doi 10 . $1016 / j . e s w a .2012 .05 .033$

Bach, N., \& Badaskar, S. (2007). A review of relation extraction. Literature review for Language and Statistics II, .

Bekoulis, G., Deleu, J., Demeester, T., \& Develder, C. (2017). Reconstructing the house from the ad: Structured prediction on real estate classifieds. In Proceedings of the 15th Conference of the European Chapter of the Association for Computational Linguistics: (Volume 2, Short Papers) (pp. 274-279). Valencia, Spain.

Bengio, Y., Simard, P., \& Frasconi, P. (1994). Learning long-term dependencies with gradient descent is difficult. Transactions on neural networks, 5(2), 157-166. doi:10.1109/72.279181

Bohnet, B., \& Nivre, J. (2012). A transition-based system for joint part-of-speech tagging and labeled non-projective dependency parsing. In Proceedings of the 2012 
Joint Conference on Empirical Methods in Natural Language Processing and Computational Natural Language Learning (pp. 1455-1465). Jeju Island, Korea: Association for Computational Linguistics.

Carreras, X. (2007). Experiments with a higher-order projective dependency parser. In Proceedings of the 2007 Joint Conference on Empirical Methods in Natural Language Processing and Computational Natural Language Learning (pp. 957-961). Prague, Czech: Association for Computational Linguistics.

Caruana, R., Lawrence, S., \& Giles, L. (2000). Overfitting in neural nets: Backpropagation, conjugate gradient, and early stopping. In Proceedings of the 13th International Conference on Neural Information Processing Systems (pp. 381-387). Denver, USA: MIT Press.

Chen, D., \& Manning, C. (2014). A fast and accurate dependency parser using neural networks. In Proceedings of the 2014 Conference on Empirical Methods in Natural Language Processing (pp. 740-750). Doha, Qatar: Association for Computational Linguistics.

Chiu, J., \& Nichols, E. (2016). Named entity recognition with bidirectional LSTMCNNs. Transactions of the Association for Computational Linguistics, 4, 357-370.

Chu, Y.-J., \& Liu, T.-H. (1965). On shortest arborescence of a directed graph. Scientia Sinica, 14, 1396-1400.

Collobert, R., Weston, J., Bottou, L., Karlen, M., Kavukcuoglu, K., \& Kuksa, P. (2011). Natural language processing (almost) from scratch. Journal of Machine Learning Research, 12, 2493-2537.

Daumé III, H., Langford, J., \& Marcu, D. (2009). Search-based struca tured prediction. Machine Learning Journal, 75(3), 297-325. doi:10.1007/ s10994-009-5106-x.

Dozat, T., \& Manning, C. D. (2017). Deep biaffine attention for neural dependency 700 parsing. In Proceedings of the International Conference for Learning Representations (pp. 1-8). Toulon, France. 
Dyer, C., Ballesteros, M., Ling, W., Matthews, A., \& Smith, N. A. (2015). Transitionbased dependency parsing with stack long short-term memory. In Proceedings of the 53rd Annual Meeting of the Association for Computational Linguistics and the 7th International Joint Conference on Natural Language Processing (Volume 1: Long Papers) (pp. 334-343). Beijing, China.

Edmonds, J. (1967). Optimum branchings. Journal of research of the National Bureau of Standards, 71B(4), 233-240.

Eisner, J. M. (1996). Three new probabilistic models for dependency parsing: An exploration. In Proceedings of the 16th International Conference on Computational Linguistics (Volume 1) (pp. 340-345). Copenhagen, Denmark.

Fundel, K., Kffner, R., \& Zimmer, R. (2007). Relex-relation extraction usq ing dependency parse trees. Bioinformatics, 23(3), 365-371. doi:10.1093/ bioinformatics/bt1616.

715 Gillick, D., Brunk, C., Vinyals, O., \& Subramanya, A. (2016). Multilingual language processing from bytes. In Proceedings of the 2016 Conference of the North American Chapter of the Association for Computational Linguistics: Human Language Technologies (pp. 1296-1306). San Diego, California.

Gilmer, J., Schoenholz, S. S., Riley, P. F., Vinyals, O., \& Dahl, G. E. (2017). Neural message passing for quantum chemistry. arXiv preprint arXiv:1704.01212, .

Goldberg, Y., \& Hirst, G. (2017). Neural Network Methods in Natural Language Processing. Morgan \& Claypool Publishers.

Goodfellow, I., Bengio, Y., \& Courville, A. (2016). Deep Learning. MIT Press. http : //www.deeplearningbook.org

725 Graves, A., r. Mohamed, A., \& Hinton, G. (2013). Speech recognition with deep recurrent neural networks. In Proceedings of the International Conference on Acoustics, Speech and Signal Processing (pp. 6645-6649). Vancouver, Canada. doi:10.1109/ICASSP . 2013.6638947. 
Gurulingappa, H., MateenRajpu, A., \& Toldo, L. (2012). Extraction of potential adverse drug events from medical case reports. Journal of Biomedical Semantics, 3(1), 1-15. doi:10.1186/2041-1480-3-15.

Hochreiter, S., \& Schmidhuber, J. (1997). Long short-term memory. Neural computation, 9(8), 1735-1780. doi:10.1162/neco.1997.9.8.1735.

Huang, Z., Xu, W., \& Yu, K. (2015). Bidirectional LSTM-CRF models for sequence tagging. arXiv preprint arXiv:1508.01991, .

Jaeger, H. (2001). The echo state approach to analysing and training recurrent neural networks-with an erratum note'. Bonn, Germany: German National Research Center for Information Technology GMD Technical Report, 148(34), 13.

Jung, J. J. (2012). Online named entity recognition method for microtexts in social networking services: A case study of twitter. Expert Systems with Applications, 39(9), 8066 - 8070. doi $10.1016 /$ j.eswa.2012.01.136

Kate, R. J., \& Mooney, R. (2010). Joint entity and relation extraction using cardpyramid parsing. In Proceedings of the 14th Conference on Computational Natural Language Learning (pp. 203-212). Uppsala, Sweden: Association for Computational Linguistics.

Kingma, D., \& Ba, J. (2014). Adam: A method for stochastic optimization. arXiv preprint arXiv:1412.6980, .

Kiperwasser, E., \& Goldberg, Y. (2016). Simple and accurate dependency parsing using bidirectional lstm feature representations. Transactions of the Association for Computational Linguistics, 4, 313-327.

Konkol, M., Brychen, T., \& Konopk, M. (2015). Latent semantics in named entity 口 recognition. Expert Systems with Applications, 42(7), 3470 - 3479. doi:10.1016/ j.eswa.2014.12.015.

Koo, T., Globerson, A., Carreras, X., \& Collins, M. (2007). Structured prediction models via the Matrix-Tree Theorem. In Proceedings of the 2007 Joint Conference 
on Empirical Methods in Natural Language Processing and Computational Natural Language Learning (pp. 141-150). Prague, Czech: Association for Computational Linguistics.

Küçük, D., \& Yazıc1, A. (2012). A hybrid named entity recognizer for turkish. Expert

口 Systems with Applications, 39(3), 2733 - 2742. doi:10.1016/j.eswa.2011. 08.131

Lafferty, J., McCallum, A., \& Pereira, F. (2001). Conditional random fields: Probabilistic models for segmenting and labeling sequence data. In Proceedings of the 18th International Conference on Machine Learning (pp. 282-289). San Francisco, USA: Morgan Kaufmann.

Lample, G., Ballesteros, M., Subramanian, S., Kawakami, K., \& Dyer, C. (2016). Neural architectures for named entity recognition. In Proceedings of the 2016 Conference of the North American Chapter of the Association for Computational Linguistics: Human Language Technologies (pp. 260-270). San Diego, California.

LeCun, Y., Boser, B., Denker, J. S., Henderson, D., Howard, R. E., Hubbard, W., \& Jackel, L. D. (1989). Backpropagation applied to handwritten zip code recognition. Neural Computation, 1(4), 541-551. doi $10.1162 /$ neco.1989.1.4.541.

Li, F., Zhang, M., Fu, G., \& Ji, D. (2017). A neural joint model for entity and relation 口 extraction from biomedical text. BMC Bioinformatics, 18(1), 1-11. doi:10.1186/ 775 S12859-017-1609-9.

Li, F., Zhang, Y., Zhang, M., \& Ji, D. (2016). Joint models for extracting adverse drug events from biomedical text. In Proceedings of the Twenty-Fifth International Joint Conference on Artificial Intelligence (pp. 2838-2844). New York, USA: IJCAI/AAAI Press.

Li, Q., \& Ji, H. (2014). Incremental joint extraction of entity mentions and relations. In Proceedings of the 52nd Annual Meeting of the Association for Computational Linguistics (Volume 1: Long Papers) (pp. 402-412). Baltimore, USA. 
Luong, T., Pham, H., \& Manning, C. D. (2015). Effective approaches to attentionbased neural machine translation. In Proceedings of the 2015 Conference on Empirical Methods in Natural Language Processing (pp. 1412-1421). Lisbon, Portugal: Association for Computational Linguistics.

Ma, X., \& Hovy, E. (2016). End-to-end sequence labeling via bi-directional LSTMCNNs-CRF. In Proceedings of the 54th Annual Meeting of the Association for Computational Linguistics (Volume 1: Long Papers) (pp. 1064-1074). Berlin, Germany.

McDonald, R., \& Pereira, F. (2007). Online learning of approximate dependency parsing algorithms. In Proceedings of the 11th Conference of the European Chapter of the Association for Computational Linguistics (pp. 81-88). Trento, Italy.

McDonald, R., Pereira, F., Ribarov, K., \& Hajic, J. (2005). Non-projective dependency parsing using spanning tree algorithms. In Proceedings of Human Language Technology Conference and Conference on Empirical Methods in Natural Language Processing (pp. 523-530). Vancouver, British Columbia, Canada: Association for Computational Linguistics.

Mikolov, T., Sutskever, I., Chen, K., Corrado, G. S., \& Dean, J. (2013). Distributed representations of words and phrases and their compositionality. In Proceedings of the 26th International Conference on Neural Information Processing Systems (pp. 3111-3119). Nevada, United States: Curran Associates, Inc.

Miwa, M., \& Bansal, M. (2016). End-to-end relation extraction using LSTMs on sequences and tree structures. In Proceedings of the 54th Annual Meeting of the Association for Computational Linguistics (Volume 1: Long Papers) (pp. 1105-1116). Berlin, Germany.

Miwa, M., \& Sasaki, Y. (2014). Modeling joint entity and relation extraction with table representation. In Proceedings of the 2014 Conference on Empirical Methods in Natural Language Processing (pp. 1858-1869). Doha, Qatar: Association for Computational Linguistics. 
Nadeau, D., \& Sekine, S. (2007). A survey of named entity recognition and classification. Lingvisticae Investigationes, 30(1), 3-26. doi $10.1075 / 1$ i.30.1.03nad.

Nagaraja, C. H., Brown, L. D., \& Zhao, L. H. (2011). An autoregressive approach

n to house price modeling. The Annals of Applied Statistics, 5(1), 124-149. doi 10 . $1214 / 10-$ AOAS380.

Nguyen, N., \& Guo, Y. (2007). Comparisons of sequence labeling algorithms and extensions. In Proceedings of the 24th International Conference on Machine Learning (pp. 681-688). Corvallis, USA: ACM. doi 10 .1145/1273496.1273582.

Nivre, J. (2003). An efficient algorithm for projective dependency parsing. In Proceedings of the 8th International Workshop on Parsing Technologies (pp. 149-160).

820 Nancy, France.

Nivre, J. (2009). Non-projective dependency parsing in expected linear time. In Proceedings of the Joint Conference of the 47th Annual Meeting of the Association for Computational Linguistics and the 4th International Joint Conference on Natural Language Processing of the Asian Federation of Natural Language Processing (pp. 351-359). Singapore.

Nivre, J., Hall, J., Nilsson, J., Eryiğit, G., \& Marinov, S. (2006). Labeled pseudoprojective dependency parsing with support vector machines. In Proceedings of the 10th Conference on Computational Natural Language Learning (pp. 221-225). New York, USA: Association for Computational Linguistics.

Pace, K., Barry, R., Gilley, O. W., \& Sirmans, C. (2000). A method for spatialtemporal forecasting with an application to real estate prices. International Journal of Forecasting, 16(2), 229 - 246. doi:10.1016/S0169-2070 (99)00047-3.

Pascanu, R., Mikolov, T., \& Bengio, Y. (2013). On the difficulty of training recurrent neural networks. In Proceedings of the 30th International Conference on International Conference on Machine Learning (pp. 1310-1318). Atlanta, USA: JMLR.org. 
Peng, F., \& McCallum, A. (2006). Information extraction from research papers using conditional random fields. Information processing \& management, 42(4), 963-979. doi:10.1016/j.ipm.2005.09.002?

Rabiner, L., \& Juang, B. (1986). An introduction to hidden markov models. IEEE ASSP Magazine, 3(1), 4-16. doi:10.1109/MASSP .1986.1165342

Ratinov, L., \& Roth, D. (2009). Design challenges and misconceptions in named entity recognition. In Proceedings of the 13th Conference on Computational Natural Language Learning (pp. 147-155). Boulder, USA: Association for Computational Linguistics.

dos Santos, C., Xiang, B., \& Zhou, B. (2015). Classifying relations by ranking with convolutional neural networks. In Proceedings of the 53rd Annual Meeting of the Association for Computational Linguistics and the 7th International Joint Conference on Natural Language Processing (Volume 1: Long Papers) (pp. 626-634). Beijing, China.

Socher, R., Chen, D., Manning, C. D., \& Ng, A. (2013). Reasoning with neural tensor networks for knowledge base completion. In Proceedings of the 26th International Conference on Neural Information Processing Systems (pp. 926-934). Nevada, United States: Curran Associates, Inc.

Srivastava, N., Hinton, G., Krizhevsky, A., Sutskever, I., \& Salakhutdinov, R. (2014). Dropout: A simple way to prevent neural networks from overfitting. Journal of Machine Learning Research, 15(1), 1929-1958.

Sutskever, I., Vinyals, O., \& Le, Q. V. (2014). Sequence to sequence learning with neural networks. In Proceedings of the 27th International Conference on Neural Information Processing Systems (pp. 3104-3112). Montreal, Canada: MIT Press.

Taskar, B., Guestrin, C., \& Koller, D. (2003). Max-margin markov networks. In Proceedings of the 16th International Conference on Neural Information Processing Systems (pp. 25-32). Bangkok, Thailand: MIT Press. 
Tsochantaridis, I., Hofmann, T., Joachims, T., \& Altun, Y. (2004). Support vector machine learning for interdependent and structured output spaces. In Proceedings of the 21st International Conference on Machine Learning (pp. 104-112). Helsinki, Finland: ACM. doi:10.1145/1015330.1015341.

Tutte, W. T. (2001). Graph theory. In Encyclopedia of Mathematics and its Applications (p. 138). Cambridge University Press volume 21.

Vinyals, O., Fortunato, M., \& Jaitly, N. (2015). Pointer networks. In Proceedings of the 28th International Conference on Neural Information Processing Systems (pp. 2692-2700). Montreal, Canada: Curran Associates, Inc.

Wang, D., \& Li, M. (2017). Stochastic configuration networks: Fundamentals and

1 algorithms. IEEE Transactions on Cybernetics, 47(10), 3466-3479. doi:10 .1109/ TCYB.2017.2734043.

Wang, W., \& Chang, B. (2016). Graph-based dependency parsing with bidirectional 1stm. In Proceedings of the 54th Annual Meeting of the Association for Computational Linguistics (Volume 1: Long Papers) (pp. 2306-2315). Berlin, Germany.

Xu, Y., Mou, L., Li, G., Chen, Y., Peng, H., \& Jin, Z. (2015). Classifying relations via long short term memory networks along shortest dependency paths. In Proceedings of the 2015 Conference on Empirical Methods in Natural Language Processing (pp. 1785-1794). Lisbon, Portugal: Association for Computational Linguistics.

Yamada, H., \& Matsumoto, Y. (2003). Statistical dependency analysis with support vector machines. In Proceedings of the 8th International Workshop on Parsing Technologies (pp. 195-206). Nancy, France.

Zhang, H., \& McDonald, R. (2012). Generalized higher-order dependency parsing with cube pruning. In Proceedings of the 2012 Joint Conference on Empirical Methods in Natural Language Processing and Computational Natural Language Learning (pp. 320-331). Jeju Island, Korea: Association for Computational Linguistics.

Zhang, X., Cheng, J., \& Lapata, M. (2017). Dependency parsing as head selection. In Proceedings of the 15th Conference of the European Chapter of the Association 
for Computational Linguistics: (Volume 1, Long Papers) (pp. 665-676). Valencia, Spain.

Zheng, S., Hao, Y., Lu, D., Bao, H., Xu, J., Hao, H., \& Xu, B. (2017). Joint entity and relation extraction based on a hybrid neural network. Neurocomputing, 257, 59 66. doi:10.1016/j.neucom.2016.12.075. 